Check for updates

Cite this: RSC Adv., 2018, 8, 3941

Received 2nd November 2017 Accepted 26th December 2017

DOI: 10.1039/c7ra12070b

rsc.li/rsc-advances

\section{Antioxidative and antimicrobial edible chitosan films blended with stem, leaf and seed extracts of Pistacia terebinthus for active food packaging}

Murat Kaya, (D) *a Sanaz Khadem, ${ }^{\text {ab }}$ Yavuz Selim Cakmak, ${ }^{a}$ Muhammad Mujtaba, (D) as Sedef Ilk, ${ }^{\mathrm{C}}$ Lalehan Akyuz, (D) ${ }^{\mathrm{d}}$ Asier M. Salaberria, ${ }^{\mathrm{e}}$ Jalel Labidi, ${ }^{\mathrm{D}}{ }^{\mathrm{e}}$ Ako Hamasaeed Abdulqadir ${ }^{a}$ and Engin Deligöz ${ }^{f}$

\begin{abstract}
Methanol extracts of stem, leaf, and seed obtained from Pistacia terebinthus which are rich in phenolic compounds were used for the first time to produce chitosan-based antioxidative and antimicrobial films. All the produced films were characterized by FT-IR, TGA, DSC, SEM, contact angle measurements, and UV-Vis spectroscopy and mechanically. As was expected, incorporation of the plant extracts into chitosan films enhanced the antioxidant and antimicrobial activities effectively. Also, the elasticity of chitosan-seed and chitosan-stem films was improved. The eco-friendly nature of the produced blend films was determined through soil and water degradation analyses. All these findings lead to the conclusion that the produced blend films with Pistacia terebinthus extracts can be applied as alternative food packaging material.
\end{abstract}

\section{Introduction}

Petroleum-based films are creating serious environmental problems worldwide due to their un-degradable features and harmful effects on humans and other organisms. ${ }^{\mathbf{1} 2}$ To escape these problems, scientists have been developing biodegradable and edible films using natural ingredients such as lipids, proteins, polysaccharides and some other animal and plant extracts. ${ }^{3-7}$ Edible films improve the shelf life of products by delaying food spoilage, preserving food against pathogens, preventing oxidation and protecting from moisture. ${ }^{3,8,9}$ The natural materials used for the production of edible films have many benefits such as edibility, biocompatibility, antioxidant, thermostability and antimicrobial properties, food protection,

${ }^{a}$ Department of Biotechnology and Molecular Biology, Faculty of Science and Letters, Aksaray University, 68100 Aksaray, Turkey. E-mail: muratkaya3806@yahoo.com; Fax: +90-382-288-2125; Tel: +90-382-288-2184

${ }^{b}$ University of Science and Culture, The Faculty of Basic Sciences and Technologies, 19166, Tehran, Iran

${ }^{c}$ Central Laboratory Research Center, Ömer Halisdemir University, 51240 Niğde, Turkey

${ }^{d}$ Aksaray University, Technical Vocational School, Department of Chemistry Technology, 68100, Aksaray, Turkey

${ }^{e}$ Biorefinery Processes Research Group, Department of Chemical and Environmental Engineering, University of the Basque Country (UPV/EHU), Plaza Europa 1, 20018 Donostia-San Sebastian, Spain

${ }^{f}$ Department of Physics, Faculty of Science and Letters, Aksaray University, 68100 Aksaray, Turkey

${ }^{g}$ Institute of Biotechnology, Ankara University, Ankara 06110, Turkey pharmaceutical applications etc. Also, these materials are inexpensive, easily available and abundant.

Chitosan, a deacetylated derivative of chitin is a well-known commonly used film forming material. ${ }^{10}$ Chemically chitosan consists of $(1,4)$-linked 2-amino-deoxy- $b$-D-glucan. It has a broad spectrum of applications in every practical field of life directly or indirectly i.e. pharmacy, medicine, agriculture, food, cosmetic, textile and water treatment etc. thanks to it's acceptable mechanical, chemical and biological properties. ${ }^{11-13}$ To improve the mechanical, hydrophobic, antioxidant and antimicrobial properties of native chitosan films, various active compounds (lipids, fatty acids, essential oils and some other plant extracts) have been incorporated into chitosan films. ${ }^{\mathbf{1 4 , 1 5}}$

P. terebinthus is a deciduous tree and belongs to Anacardiaceae family. It is a small tree growing up to $10 \mathrm{~m}$ in the Middle East and Mediterranean region. ${ }^{\mathbf{1 6}}$ The extracts of this plant have anti-inflammation and antioxidant flavonoid, omega-3 fatty acids, tannin and other valuable compounds. ${ }^{17,18}$ Being a traditional medicine, it is using for the treatment of liver and kidney and also drinks coffee. ${ }^{\mathbf{1 9 2 0}}$ It can be used in the production of vegetable oils because of its high amount of oleic acid. ${ }^{17}$ These advantages make the extracts of $P$. terebinthus as a suitable ingredient to be used in edible film production.

Considering various useful properties of $P$. terebinthus, this study was aimed to produce edible chitosan-based films incorporated with the stem, leaf, and seed methanol extracts. The produced films were characterized both biologically (antimicrobial and antioxidant activities) and physicochemically (FTIR, TGA, DSC, SEM, contact angle, mechanical properties and optical transmittance). 


\section{Materials and methods}

\subsection{Materials}

Chitosan (medium molecular weight and 85\% de-acetylation degree, Cas No. 448877) was purchased from SIGMA-Aldrich, USA. The acetic acid $\left(\mathrm{CH}_{3} \mathrm{COOH}\right)$, hydrochloric acid $(\mathrm{HCl})$, glycerol $\left(\mathrm{C}_{3} \mathrm{H}_{8} \mathrm{O}_{3}\right)$ and methanol $\left(\mathrm{CH}_{3} \mathrm{OH}\right)$ were supplied from MERCK, Germany. The plant material (P. terebinthus) was collected from Aleppo, Northern Iraq on 13 July 2016.

\subsection{Extract isolation}

Each plant part i.e., seed, leaf, and stem were ground to fine powder by using WARING commercial blender. Methanol was added to each sample and sonicated using WUC-D10H, DAIHAN sonicator for $10 \mathrm{~m}$. Finally, the methanol solution was evaporated and plant extracts were obtained.

\subsection{Determination of phenolic compounds}

The phenolic component analysis was performed on an Agilent brand 1290 Infinity model HPLC instrument. Analyzes were performed using the C18 column and DAD detector according to Caponio, et al. ${ }^{21}$ \% acetic acid (A) and methanol (B) were

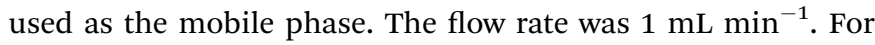
mobile phase; ( 3 min 95\% A/5\% B, 15 min 80\% A/20\% B, 2 min isocratic, $10 \mathrm{~min} 60 \% \mathrm{~A} / 40 \% \mathrm{~B}, 10 \mathrm{~min} 50 \% 10 \mathrm{~min}$ ) was applied with a gradient of $100 \% \mathrm{~B}$. The extract was injected with $10 \mu \mathrm{L}$ of the solution prepared with methanol to give a concentration of $20 \mathrm{mg} \mathrm{mL}^{-1}$.

\subsection{Film production}

Four different types of chitosan films were produced, three of which were incorporated with leaf, stem and seed extract while the fourth one was used as a control without incorporating any extract. Briefly, $1 \mathrm{~g}$ of chitosan was added to $100 \mathrm{~mL}$ of distilled water and $100 \mu \mathrm{L}$ of $1 \%$ acetic acid to make chitosan solution. The mixture was stirred for $48 \mathrm{~h}$ via magnetic stirrer. Then $1 \mathrm{~g}$ of each plant extract was added to $5 \mathrm{~mL}$ methanol and was vortex (VELP SCIENTIFICA, Vortex mixer) for $5 \mathrm{~min}$. Then $1 \mathrm{~mL}$ of methanol mixture was incorporated with $60 \mathrm{~mL}$ of chitosan solution and $100 \mu \mathrm{L}$ of glycerol as plasticizer. All solutions were homogenized at $26000 \mathrm{rpm}$ for $5 \mathrm{~min}$ with a homogenizer (Heidolph, Silent Crusher M). Each solution was poured into a plastic Petri dish. The Petri dishes were kept at $30^{\circ} \mathrm{C}$ for $48 \mathrm{~h}$. Finally, the films were collected from Petri dishes and stored for further analysis.

\subsection{Fourier transform infrared spectroscopy (FT-IR)}

The FT-IR spectra of control and blend films were recorded at $8 \mathrm{~cm}^{-1}$ resolution and in the range between 600 to $4000 \mathrm{~cm}^{-1}$ by PerkinElmer Spectrometer.

\subsection{Thermogravimetric analysis (TGA)}

The thermal stabilities of the films were carried out for both control and blend films via TGA Q500 - TA Instruments. 5 mg of the film sample was used for each analysis. Film samples were heated at a constant temperature rate of $10{ }^{\circ} \mathrm{C} \mathrm{min}^{-1}$ from $30{ }^{\circ} \mathrm{C}$ to $600{ }^{\circ} \mathrm{C}$ under a nitrogen atmosphere.

\subsection{Differential scanning calorimeter (DSC)}

To determine the endothermic and exothermic properties of film samples incorporated with $P$. terebinthus extracts, DSC analysis was carried by using a Mettler Toledo DSC822e (Schwerzenbach, Switzerland). The analysis was carried out under $\mathrm{N}_{2}$ atmosphere under the temperature range between -50 to $420{ }^{\circ} \mathrm{C}$. For each film sample, $50 \mathrm{mg}$ was used during the analysis. Film samples were placed in a hermetic aluminum pan and the heating scan was regulated as $5{ }^{\circ} \mathrm{C} \min ^{-1}$.

\subsection{Scanning electron microscopy (SEM)}

For determination of surface morphology and cross-section of the control film and chitosan-based films incorporated with $P$. terebinthus extracts SEM analysis was performed using Zeiss, Eco 40 (Germany). All the film samples were coated with the layer of gold/palladium prior to taking images.

\subsection{Biodegradability}

Biodegradability was tested by using two different methods i.e., water solubility and soil degradation. Both of the methods were repeated thrice. Solubility in water and soil was calculated by using the following equation:

$$
\text { WL }(\%)=\text { weight loss/initial weight } \times 100
$$

2.9.1. Solubility in water. For testing the films solubility in water, $10 \mathrm{mg}$ of each film sample was cut into small pieces $(2 \times$ $3 \mathrm{~cm}^{2}$ ) and the initial dry weights were measured for all the four samples. Next, $20 \mathrm{~mL}$ of water was added to the films and were kept at room temperature for $48 \mathrm{~h}$. After $48 \mathrm{~h}$ of immersion of the film samples, they were dried at $60{ }^{\circ} \mathrm{C}$ and were weight again.

2.9.2. Soil degradability. Small pieces of the dry films $(2 \times 3$ $\mathrm{cm}^{2}$ ) were scaled and then put into the enrichment soil. About $10 \mathrm{~mL}$ of water was added to the samples daily and after 15-30 days the final weights were measured. For having the natural biodegradation environment, we did not modify soil microflora. Chitosan and other nutrients in the samples were carbon and nitrogen sources for microbes and by adding water the soil microorganisms were activated.

\subsection{Contact angle}

Sessile drop contact angle analysis was carried out for control film and chitosan-based films incorporated with $P$. terebinthus extracts using a Data Physics video-based contact angle measurement system OCA20. Accurate drop volume was measured through software controlled dosing volume weightdrop. The contact angle was measured by using water. For each film sample, eight measurements were taken. 


\subsection{UV-Vis transparency}

The transparency of control film and chitosan-based blended with $P$. terebinthus plant extracts was observed in the visible light range (400-700 $\mathrm{nm}$ ) by using a UV spectrophotometer (Shimadzu UV-3600 UV-Vis-NIR).

\subsection{Mechanical properties}

The tensile strength (TS), the percentage of elongation at break (EB \%), and Young's modulus (YM) were recorded in the ambient temperature for control film and chitosan-based blended film with $P$. terebinthus plant extracts. The load cell of $250 \mathrm{~N}$ and at a rate of $5 \mathrm{~mm} \mathrm{~min}^{-1}$, deformation of the films were determined by using Material Testing Systems (MTS Insight 10) equipment. MTS Test Works 4 software was applied for calculating the mechanical properties. Mitutoyo, China (a digital micrometer) was used for the measurements of the film thickness.

\subsection{Antioxidant activity}

2.13.1. DPPH (2,2'-diphenyl-1-picrylhydrazyl) radical scavenging assay. UV-visible spectrometry was applied to evaluate the scavenging rate of 2,2-diphenyl-1-picrylhydrazyl (DPPH) radicals of chitosan control and chitosan blended films. Also, DPPH solution was used as a reagent by following a previous method reported by (ref. 14) with small amendments. Briefly, $10 \mathrm{mg}$ of each film sample was dissolved in $1 \mathrm{~mL}$ of DPPH solution $\left(6 \times 10^{-1} \mathrm{M}\right)$. The mixtures were incubated for $3 \mathrm{~h}$ in inky condition and then measured at $517 \mathrm{~nm}$ via a BECKMAN COULTER, DU 730 UV spectrophotometer. The analysis was repeated three times and the antioxidant activities for each sample were computed with the following formula:

$$
\begin{gathered}
\text { Inhibition }(I \%)=\left(\left(A_{\text {control }}-A_{\text {sample }}\right) / A_{\text {control }}\right) \times 100 \\
A_{\text {control }}=\text { absorbance of DPPH solution } \\
A_{\text {sample }}=\text { the absorbance of the films }+ \text { DPPH }
\end{gathered}
$$

\subsection{Antimicrobial activity}

2.14.1. Bacteria culture. The food and human-borne pathogenic microorganisms i.e., Escherichia coli ATCC 25922, Staphylococcus aureus ATCC 25923, Proteus microbilis ATCC 14153, Proteus vulgaris ATCC13315, Pseudomonas aeruginosa ATCC27853, Enterobacter aerogenes ATCC13048, Bacillus thuringiensis, Salmonella enterica serotype typhimurium SL 1344 and Streptococcus mutans ATCC 25175 were employed to evaluate the antibacterial properties of chitosan control and chitosan films blended with stem, leaf and seed extracts of $P$. terebinthus. All strains were sub-cultured on LB agar culture at $37{ }^{\circ} \mathrm{C}$ for $24 \mathrm{~h}$.

2.14.2. Disc diffusion test. Antimicrobial effectivity of $P$. terebinthus' extracts incorporated in chitosan films against the food-borne pathogens was evaluated by using disc-diffusion method following the method reported by National Committee for Clinical Laboratory Standard (NCCLS) with minute amendments. Microorganisms' susceptibility was adjusted by 0.5 McFarland as a reference standard. Microorganism culture suspension $\left(100 \mu \mathrm{L}, 10^{6}\right.$ cells per $\left.\mathrm{mL}\right)$ was swabbed onto a plate within Müeller-Hinton agar. Filter discs were placed on the Petri plate inoculated with bacteria and then, the tested samples $(20 \mu \mathrm{L})$ were loaded on the sterile discs and incubated at $37^{\circ} \mathrm{C}$ for $24 \mathrm{~h}$. The gentamicin discs $(10 \mu \mathrm{g}$ per disc) and solvent (alone) was used as a positive and negative control, respectively. All experiments were repeated thrice. The results were expressed as the mean diameter of inhibition zone in $\mathrm{mm} \pm$ standard deviation (mean $\pm \mathrm{SD}$ ).

\subsection{Anti-quorum sensing activity}

2.15.1. Disc diffusion test. The reporter strain Chromobacterium violaceum CV026 was obtained to evaluate the anti-QS properties of chitosan films blended with extracts of $P$. terebinthus. The bacterial suspensions were sub-cultured (aerobic growth, at $30^{\circ} \mathrm{C}$ ) overnight in LB broth. Disc diffusion assay was performed to determine the QS inhibitory activity of obtained chitosan films. Firstly, signal molecules C6-HSL $\left(0.25 \mu \mathrm{g} \mathrm{mL}{ }^{-1}\right)$ was added to LB soft-agar and then poured into the Petri plates. The strain C. violaceum CV026 was inoculated onto a surface of the solidified agar. After that, sterile discs were placed onto the agar and loaded with the tested samples $(20 \mu \mathrm{L})$. Prepared plates were incubated at $30{ }^{\circ} \mathrm{C}$ for $24-30 \mathrm{~h}$. As a control material, only solvent (negative) and gentamicin (positive) were used. Halo formation on a purple background suggested that the tested chitosan films exhibited the anti-QS inhibitory effect.

2.15.2. Quantitative determination of violacein inhibition. Quantitative determination of violacein inhibition by the synthesized chitosan blend films was carried out via the flask incubation assay. LB broth inoculated with bacteria mixed with C6-HSL $(5 \mu \mathrm{M})$ and samples were incubated for $24 \mathrm{~h}$ at $30^{\circ} \mathrm{C}$. Violacein extraction was carried out as described by. ${ }^{22}$ First of all, for the precipitation of violacein, the suspension from each flask $(1 \mathrm{~mL})$ was centrifuged at $9.000 \mathrm{~g}$ for $10 \mathrm{~min}$. For solubilization of the violacein, the samples were dissolved in dimethyl sulfoxide $(1 \mathrm{~mL})$ and vortexed. The supernatant was centrifuged again and quantified at $585 \mathrm{~nm}$ using UV-Vis spectrophotometer (PG Instruments, T-60). The assay was done triplicate and the pigment inhibition percentage was calculated by the following formula as:

$$
\begin{gathered}
\% \text { Violacein inhibition }=\left(\left(\operatorname{control}\left(\mathrm{OD}_{585}\right)-\text { test }\left(\mathrm{OD}_{585}\right)\right) /\right. \\
\text { control } \left.\left(\mathrm{OD}_{585}\right)\right) \times 100
\end{gathered}
$$

\section{Results and discussion}

\subsection{FT-IR}

In order to determine the molecular interactions in the films among chitosan and leaf, seed and stem extracts of $P$. terebinthus, FT-IR analysis was carried out. FTIR spectra of chitosan, P. terebinthus extracts and blend films are shown in Fig. 1. In the first spectrum, characteristic chitosan peaks were recorded as $-\mathrm{OH}$ bonds $\left(3288.10 \mathrm{~cm}^{-1}\right), \mathrm{C}-\mathrm{H}$ aliphatic stretching vibration $\left(2873.78 \mathrm{~cm}^{-1}\right)$, amide I $\left(1651.51 \mathrm{~cm}^{-1}\right)$, amide II 


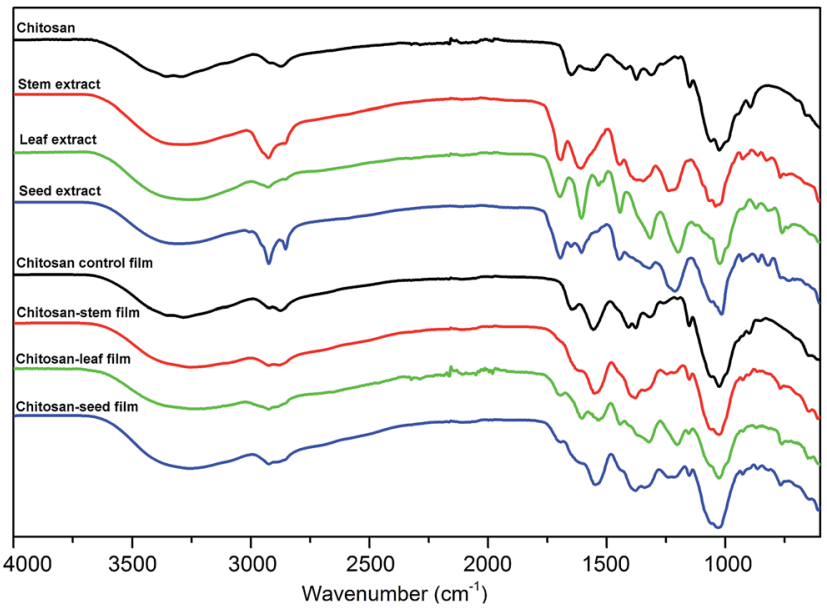

Fig. 1 FT-IR spectra of chitosan; stem, leaf and seed extracts of Pistacia terebinthus; chitosan control film; chitosan-stem film; chitosanleaf film and chitosan-seed film

(1557.79 $\left.\mathrm{cm}^{-1}\right)$, amide III $\left(1374.07 \mathrm{~cm}^{-1}\right)$ and C-O stretching vibration $\left(1025.51 \mathrm{~cm}^{-1}\right)$. As seen from the spectrum of chitosan film, structural changes occurred during the film formation. Addition of the glycerol to the film matrix (chitosan, acetic acid, and water), affected the intermolecular interactions resulted in expand in the $\mathrm{OH}$ bond and increase in the intensity of amide I, II and III bands.

The FTIR spectra of $P$. terebinthus' leaf, seed, and stem extracts showed a broad absorption peak nearly $3300 \mathrm{~cm}^{-1}$ corresponding to $-\mathrm{OH}$ stretching. However, the spectrum of the leaf extract was observed differently from the spectra of stem and seed extracts. $\mathrm{C}-\mathrm{H}$ symmetric stretching vibrations of the polyphenolic structures at around $2925.00 \mathrm{~cm}^{-1}$ showed strong absorption peaks in the seed and stem extracts, while the peak of leaf extract revealed a weak absorption in the same region. Increase in polyphenolic content in the leaf extract (see HPLC results) resulted in overlapping of the $\mathrm{OH}$ band with the $\mathrm{CH}$ stretching peak.

$\mathrm{C}=\mathrm{O}$ stretching vibrations were detected at $1691.76,1698.91$ and $1695.23 \mathrm{~cm}^{-1}$ for steam, leaf and seed extracts, respectively. Shifting of the $\mathrm{C}=\mathrm{O}$ peak absorption to lower wavelength may result from the conjugation of the carbonyl group bonded to an aromatic ring. Conjugated $\mathrm{C}=\mathrm{C}$ stretching vibrations of the aromatic ring were detected at around $1600.00 \mathrm{~cm}^{-1}$ for all the extracts. Schulz and Baranska $^{23}$ reported that the bands appearing near at $1601 \mathrm{~cm}^{-1}$ assigned to the benzene ring of the polyphenolic compounds. In the present study, these peaks of leaf and seed extract were observed at around $1606 \mathrm{~cm}^{-1}$ as more intense than the stem extract peak. This phenomenon can be explained with the leaf and seed extract had higher polyphenolic content. The peaks at around $1440.00 \mathrm{~cm}^{-1}$ can also be formed by contribution of aromatic $\mathrm{C}=\mathrm{C}$ bonds. $\mathrm{C}-\mathrm{H}$ out-ofplane bending vibrations of substitute aromatic ring were observed in the range of $927.00-750.00 \mathrm{~cm}^{-1}$.

It was observed that the intensities and frequency regions of peaks in the spectrum of chitosan film changed the type of the extract in tested samples. Inter- and intra-molecular hydrogen bonds formed between stem extract and film matrix were resulted in broader $-\mathrm{OH}$ bond peak $\left(3253.14 \mathrm{~cm}^{-1}\right)$ than in the spectrum of chitosan control film. Interestingly, $\mathrm{C}=\mathrm{O}$ stretching vibration belonging to chitosan and stem extract was not observed in the chitosan-stem film. This may be due to the fact that, all of the $\mathrm{C}=\mathrm{O}$ group found in the chitosan-stem film were affected by the hydrogen bonds. Besides, the intensities of amide II $\left(1549.12 \mathrm{~cm}^{-1}\right)$ and amide III $\left(1379.55 \mathrm{~cm}^{-1}\right)$ peaks of chitosan increased. Additionally, weak peaks observed in the wave-number range $609.00-926.27 \mathrm{~cm}^{-1}$ were attributed to $\mathrm{C}-\mathrm{H}$ bending vibrations of substituted aromatic ring. This was another indication of strong intermolecular interactions between stem extract and film matrix.

In the leaf film spectrum, - $\mathrm{OH}$ bond expanded and overlapped with $\mathrm{CH}$ stretching peak due to the hydrogen bond formation between phenolic $\mathrm{OH}$ groups and chitosan. Conjugated $\mathrm{C}=\mathrm{C}$ bonds of the aromatic ring were observed at $1605.44 \mathrm{~cm}^{-1}$ in the leaf spectrum. The intensities of the amide II $\left(1532.03 \mathrm{~cm}^{-1}\right)$, amide III $\left(1322.14 \mathrm{~cm}^{-1}\right)$ and $\mathrm{CH}_{2}$ (1204.27 $\mathrm{cm}^{-1}$ ) bending absorption increased due to the effect of the molecular interactions between leaf extract and the film matrix. Similar results were observed in the fingerprint region of the chitosan-seed film spectrum. However, $-\mathrm{OH}$ bond absorption was recorded as narrower than the leaf band absorption. This may be due to the fact that the number of hydrogen bonds was less in the chitosan-seed extract film.

Mayachiew and Devahastin ${ }^{24}$ reported that a new peak observed at the $1720 \mathrm{~cm}^{-1}$ with the addition of gooseberry extract into the chitosan film. They explained this peak as an ester linkage formed between chitosan and polyphenolic compounds. In the present study, the new peaks were observed at around $1720 \mathrm{~cm}^{-1}$ with the increase in the polyphenolic contents of extract. This result was an agreement with the Mayachiew and Devahastin result. The FTIR analysis results revealed that successfully incorporation of different extracts of $P$. terebinthus into the chitosan film resulted in different molecular interactions in the film matrix.

\subsection{TGA analysis}

The thermogravimetric results of the chitosan control and blend films are presented in Fig. 2. For all the films, degradation was recorded in three steps. The first stage was recorded around 50-100 ${ }^{\circ} \mathrm{C}$ (about $5 \%$ decomposition for each film). This first mass loss can be attributed to the water evaporation from the polymeric structures. The second step was observed around $120-200{ }^{\circ} \mathrm{C}$ and it can correspond to the decomposition of glycerol. ${ }^{25}$ The main mass loss was recorded around $240-300{ }^{\circ} \mathrm{C}$ and it was because of the denaturation of chitosan polymeric organization and plant extracts. ${ }^{26}$ In this step chitosan-stem extract film showed similar stability to the control film, however, the figure demonstrates, incorporation of seed extract to chitosan matrix decreased the thermal stability. These stages of decomposition were in agreement with the chitosan-based film incorporated with thyme oil. ${ }^{27}$ At the end of thermal degradation, ash contents for chitosan control, chitosan-stem, chitosan-leaf and chitosan-seed films was recorded as $34.44 \%$, 

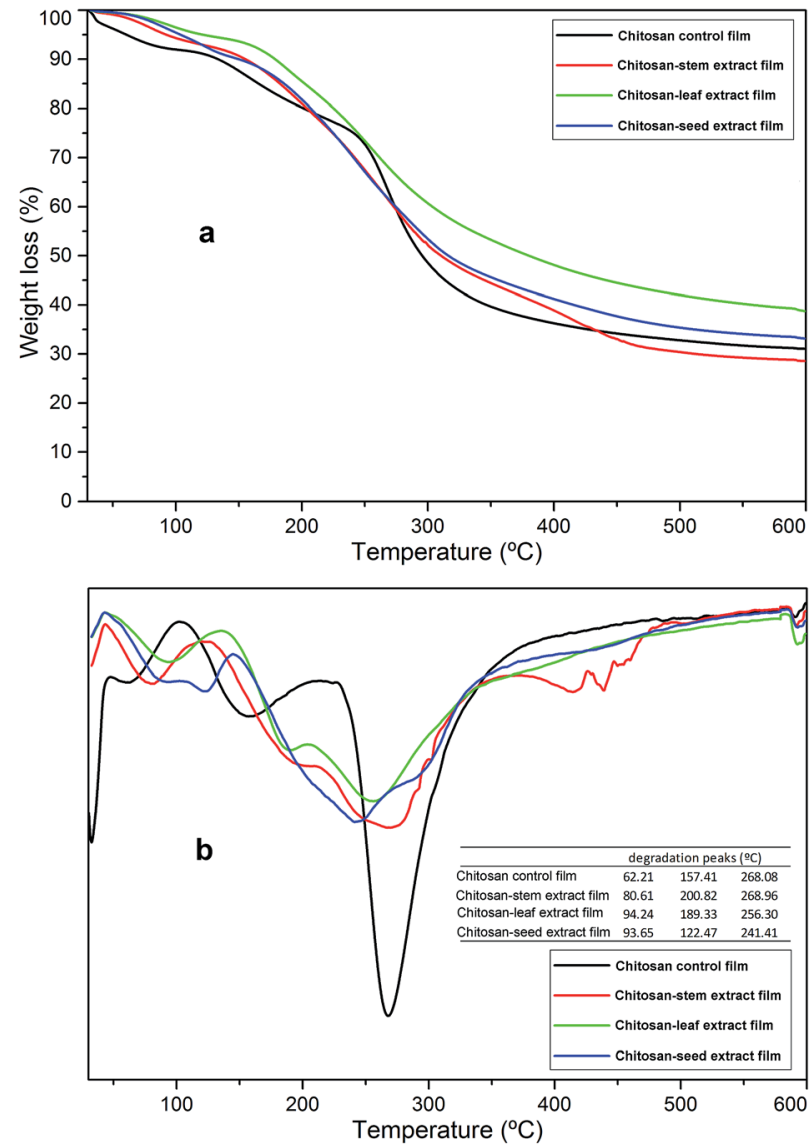

Fig. 2 (a) TGA and (b) DTG thermograms of the chitosan control film and blend films incorporated with Pistacia terebinthus' extracts as; chitosan-stem film, chitosan-leaf film and chitosan-seed film.

$29.19 \%$, 39.122 and $35.51 \%$, respectively. In general, the thermal stabilities of chitosan blend films were not affected by the incorporation of plant extracts.

Additionally considering some previous reports regarding the production of chitosan blend films, same phenomena was recorded in those reports; i.e. Pelissari, F.M. et al. ${ }^{28}$ observed the same results when cassava starch-chitosan films were incorporated with oregano essential oil. The overall thermal stability did not reveal any increase in case of blend films. Kanmani P. et al. ${ }^{29}$ reported that no alteration was observed in the thermal stability $\left(\mathrm{dTGA}_{\max }\right)$ of the chitosan films when blended with carrageenan/grapefruit seed extract.

\subsection{DSC}

The DSC thermograms of the chitosan film and composite films are shown in Fig. 3. Chitosan-control film exhibited two endothermic $\left(72.24\right.$ and $\left.154.64{ }^{\circ} \mathrm{C}\right)$ and one exothermic peak (269.65 ${ }^{\circ} \mathrm{C}$ ). The first exothermic peak at $269{ }^{\circ} \mathrm{C}$ corresponded to the phase inversion temperature of decomposition of chitosan. Peng, $\mathrm{Wu}$ and $\mathrm{Li}^{30}$ reported the similar result for chitosan film. Also, they explained that the phase inversion temperature was reduced by the addition of tea extract into the chitosan film. The similar results were found in the present study. The thermal stability of chitosan films decreased with the incorporation of

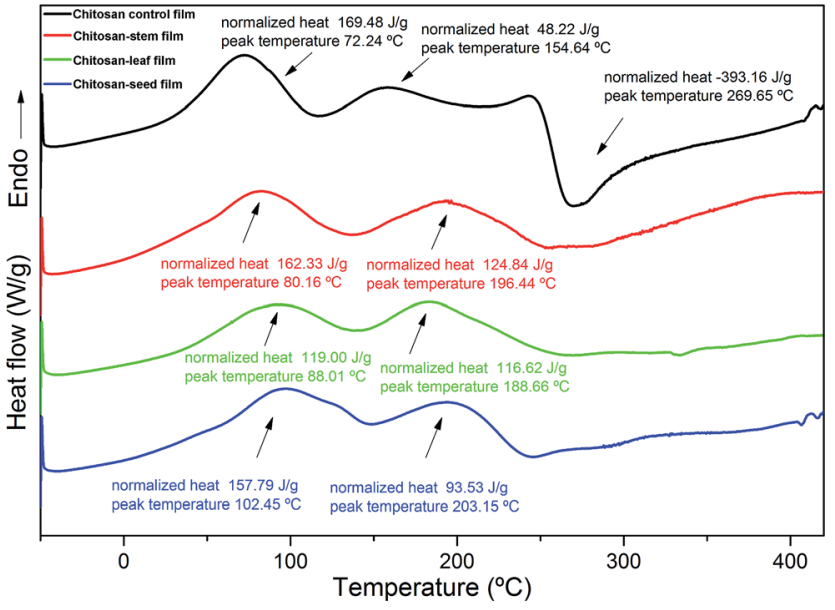

Fig. 3 DSC thermograms of the chitosan control film and blend films incorporated with Pistacia terebinthus' extracts as; chitosan-stem film, chitosan-leaf film and chitosan-seed film.

the leaf, stem and seed extracts, due to the decreasing of crystallinity of the films Martins J.T. et al. ${ }^{31}$ Blend films incorporated with $P$. terebinthus extracts showed two endothermic peaks. For chitosan-stem, chitosan-leaf and chitosan-seed the two endothermic peaks were observed as; 80.16-196.44 ${ }^{\circ} \mathrm{C}$, 88.01-186.66 ${ }^{\circ} \mathrm{C}$ and $102.45-203.15{ }^{\circ} \mathrm{C}$, respectively. The first endothermic peaks for chitosan-control and blend films can be attributed to evaporation of solvent traces (water, acetic acid, and methanol) used during the production of composite films. ${ }^{27}$ While the second endothermic DSC peaks for blend films can be ascribed to the denaturation of glycerol and extract components.

\subsection{SEM}

The surface characteristics of chitosan control and chitosan blend films are presented in Fig. 4. As is seen from the figure, surface morphology of chitosan-control was observed as smooth, compact, and without any crack or pores (Fig. 4a and b), it was similar to results of the other studies. ${ }^{25,27}$ Nevertheless, composite films exhibited irregular forms. The chitosan-stem extract film had many bulges and tiny particles on the surface (Fig. 4c and d). Chitosan-leaf extract film also exhibited heterogeneous form, but it had lower bulges and higher pieces (Fig. 4e and f) and was more homogenous than chitosan-stem extract film. Although chitosan-seed extract film showed some roughness and particles, it was detected as a more regular film to the blend films (Fig. $4 \mathrm{~g}$ and $\mathrm{h}$ ). Almost similar surface morphologies were revealed from the SEM micrographs in other studies reporting the incorporation of fruit extracts in chitosan blend films. ${ }^{32-35}$

\subsection{Water solubility and soil degradation}

The water solubility of the chitosan-control film was recorded as $24 \%$ in $48 \mathrm{~h}$. Incorporations of plant extracts into the films demonstrated significant $(P<0.05)$ increase in film solubilities as $35.27 \%, 45.31 \%$ and $50.7 \%$ for chitosan-leaf, chitosan-stem, 

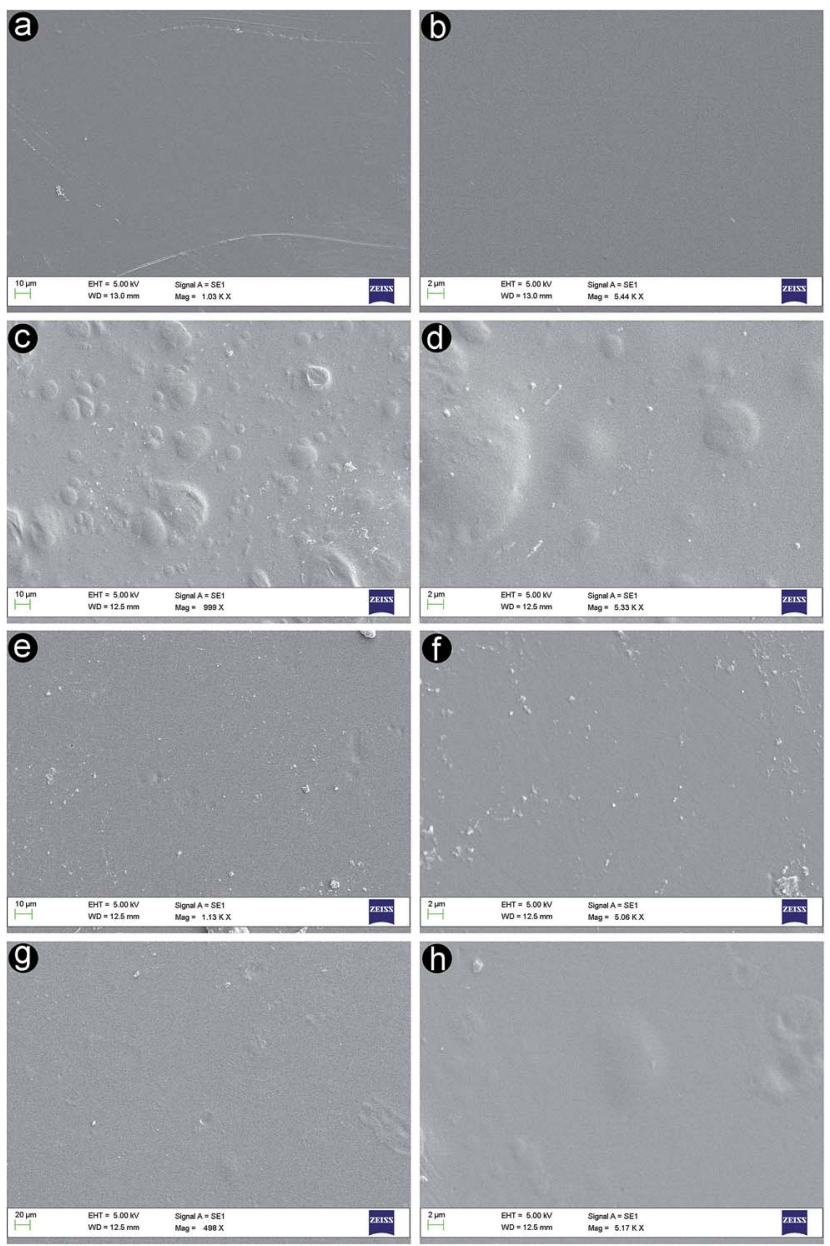

Fig. 4 Scanning electron microscopy of the chitosan control and blend films of Pistacia terebinthus' extracts: ( $a$ and b) chitosan-control film, (c and d) chitosan-stem film, (e and f) chitosan-leaf film and (g and h) chitosan-seed film.

and chitosan-seed film, respectively. This chitosan film degradation was witnessed in accordance with previous reports $(23.52$ $\pm 1.26 \%){ }^{36}$ The weak intermolecular interactions between plant extracts and chitosan matrix resulted in higher degradation values into the chitosan blend films. Higher solubility rate for the chitosan-seed film was probably due to the faster diffusion and disruption of molecular interactions.

Soil degradation rates in 15 days were recorded as $89.04 \%$ for chitosan control film, $98.76 \%$ for chitosan-leaf film, $78.65 \%$ for chitosan-stem film and $56.35 \%$ for the chitosan-seed film. The chitosan-leaf film exhibited higher soil degradability, however, lower soil degradability was observed for the chitosan-seed film.

\subsection{Contact angle}

The contact angle is another parameter to determine hydrophobic and hydrophilic nature of materials. Water wettability of the film surface is associated with the final angle of the water drop. In order to check the effect of the stem, seed and leaf extract on film wettability, the contact angle values were calculated for chitosan control, chitosan-stem, chitosan-seed and chitosan-leaf films as $86.3 \pm 1.43^{\circ}, 82.1 \pm 1.83^{\circ}$ and $91.35 \pm$ $3.05^{\circ}$ and $61.8 \pm 5.54^{\circ}$, respectively. Chitosan-leaf extract film showed hydrophilic feature as its contact angle observed at $61.8^{\circ}$. However, chitosan-leaf extract film has lower water solubility rate. As seen from the HPLC results, leaf extract has maximum polyphenolic content. Increase in polyphenolic content caused strong interactions in the film matrix and reduced water solubility. However, hydrophilic groups of the polyphenolic compounds such as carboxyl, hydroxyl were interacted with the water on the film surface and reduced the contact angle. Same phenomena were reported by Jin et al. ${ }^{37}$ when chitosan matrix was incorporated with genipin. An increase in the genipin content affected the overall film wettability in a positive manner. In another report by Ramírez et al. ${ }^{38}$ a reducing pattern was observed for contact angle of the chitosan-based when incorporated with murta leaves extract.

\subsection{Visual appearance and light transmission of the films}

Appearance and color of the food coatings are one of the most important factors influencing the nutrients quality and customers attraction. As is shown in the Fig. 5 chitosan control film was appeared as clear, glossy, smooth, and without any tents. The visual appearance of chitosan-stem extract, chitosanleaf extract, and chitosan-seed extract composite films was observed as light yellow, green and light brown color, respectively. Previous literature reports also, explained the same phenomena, that adding plant extracts to chitosan matrix, changed the film's color. ${ }^{27,39}$ Control, chitosan-stem extract, and chitosan-seed extract films were homogeneous and without any wrinkle, pores or bubbles. Contrarily, chitosan-leaf extract film exhibited granules and evenly distributed rare bubbles on its

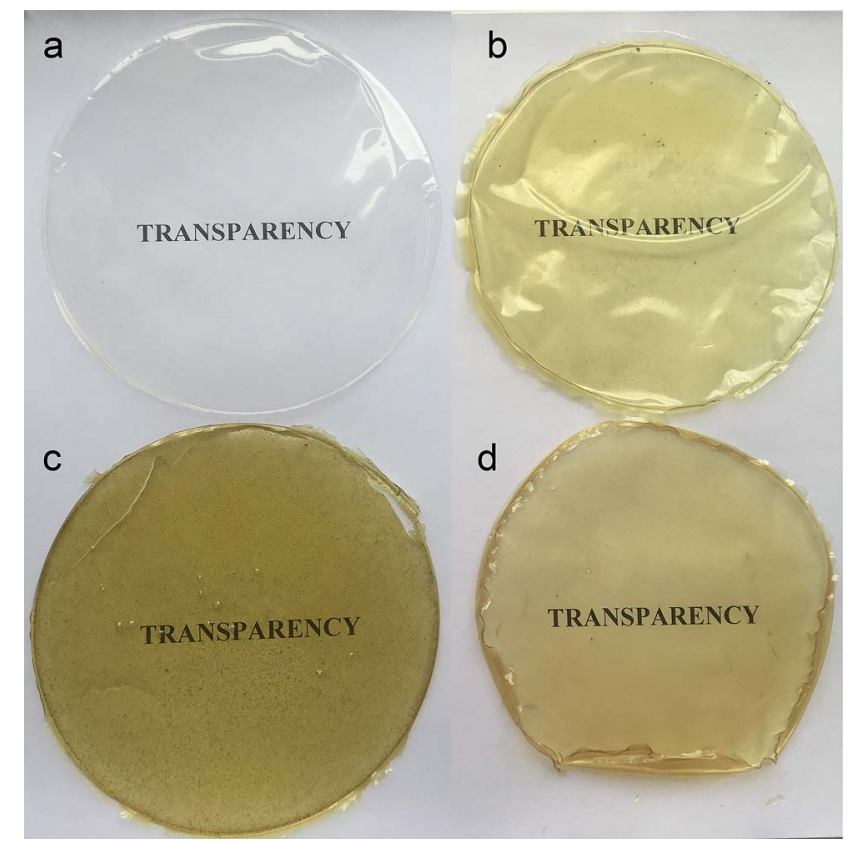

Fig. 5 Visual transparency of the chitosan control film and blend films incorporated with Pistacia terebinthus' extracts as; chitosan-stem film, chitosan-leaf film and chitosan-seed film. 
surface. Chitosan-leaf film had not enough flexibility and it was broken easily. The color of chitosan-leaf extract film can be due to different compounds in the leaf structure and existence more pigments (chloroplasts) than the other parts.

Optical transmittance of chitosan control and blend films was measured in the visible spectrum (Fig. 6). The addition of plant extracts into chitosan film decreased the transparency. The transmittance rate at $700 \mathrm{~nm}$ was recorded $89.79 \%$ for chitosan control film, $66.6 \%$ for chitosan-seed film, $63.16 \%$ for chitosan-stem film and $44.76 \%$ for the chitosan-leaf film. The gradual decrease in the transparency rate of blend films can possibly due to the presence of pigments and tints on the film surface.

\subsection{Mechanical properties and thickness}

Evaluation of tensile strength, Young's modulus and elongation at break are vital characteristics for understanding the behavior of biomaterials during their practical applications i.e., packaging and shipping. ${ }^{40}$ In this context, mechanical properties of blend films were measured and the results are given in Fig. 7 . Young's modulus can be described as a measurement of the stiffness of any bio-based materials. On the other hand, the tensile strength can be explained as the maximum engineering stress level reached in a tension test. When the value of Young's modulus is large, it indicates the stiffness of the material. In this context, in the present study, due to the higher value of Young's modulus for chitosan control and chitosan-leaf films were observed comparatively stiffer. For chitosan control film, Young's modulus (1251.89 $\pm 33.90 \mathrm{MPa})$ and tensile strength $(26.86 \pm 3.50 \mathrm{MPa})$ were recorded about two times higher than chitosan-leaf extract film and much higher than chitosan-stem extract and chitosan-seed extract films. In this context, chitosan control and chitosan-leaf films were recorded stiffer than other films.

From Fig. 7, it can be seen that chitosan-stem extract and chitosan-seed extract films exhibited a large elongation at break. Therefore, these composite films had ductile nature. On

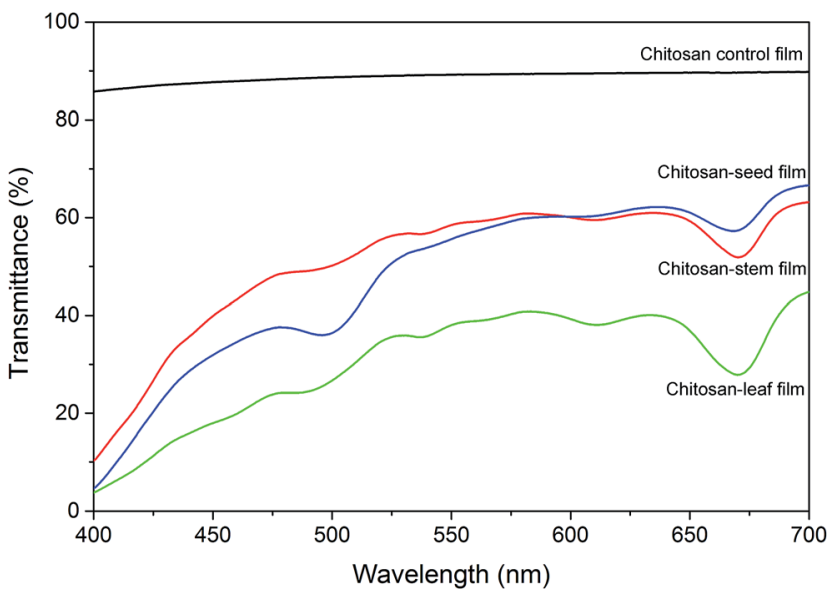

Fig. 6 Optical transmittance of the chitosan control film and blend films incorporated with Pistacia terebinthus' extracts as; chitosan-stem film, chitosan-leaf film and chitosan-seed film.
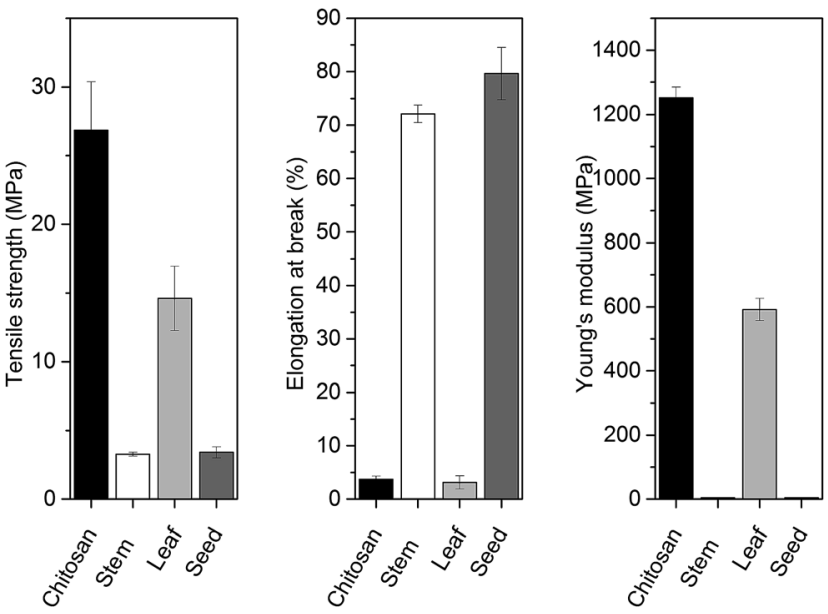

Fig. 7 The mechanical properties of the chitosan control film and blend films incorporated with Pistacia terebinthus' extracts as; chitosan-stem film, chitosan-leaf film and chitosan-seed film.

the other hand, elongation at break values of chitosan-stem extract $(72.13 \pm 1.65 \%)$ and chitosan-seed extract $(79.62 \pm$ $4.86 \%)$ films was observed much higher than chitosan control film $(3.72 \pm 0.57 \%)$ and chitosan-leaf extract film (3.11 \pm $1.23 \%)$. These results exhibited that incorporation of seed and stem extract from P. terebinthus highly increased the elasticity of the chitosan film. So the chitosan control film can be suggested as the stiffest among the other considered films. It can be noted that tensile strength and Young's modulus of chitosan control film were recorded about two times higher than chitosan-leaf extract film and much higher than chitosan-stem extract and chitosan-seed extract films. It is evident that the chitosan-stem extract exhibited flexible and weak behavior as indicated by a very high elongation at break, a very low tensile strength $(3.28$ $\pm 0.15 \mathrm{MPa}$ ). These results also revealed that incorporation of stem and seed extract from $P$. terebinthus highly increased the elasticity of the chitosan film.

When examined visually and handled, it was realized that chitosan-leaf extract film was fragile. However, chitosan-stem extract and chitosan-seed extract films were flexible. In some earlier studies, the addition of essential oils and plant extracts into the chitosan films increased the elongation at break $\mathrm{k}^{39,41,42}$ as it was recorded in the present. Park and $\mathrm{Zhao}^{43}$ reported the reduction in tensile strength and elongation at break due to the decreasing of the crystallinity of the edible film and intermolecular hydrogen bonding formed in the film matrix. As seen from the HPLC results, the polyphenolic content of the leaf extract was too high, which capable of the $\mathrm{H}$ bonding.

Thickness values of chitosan control, chitosan-stem, chitosan-leaf and chitosan-seed films were measured as $50 \pm$ $9.4 \mu \mathrm{m}, 71.75 \pm 5.31 \mu \mathrm{m}, 104.75 \pm 14.3 \mu \mathrm{m}$ and $70.8 \pm 1.7 \mu \mathrm{m}$, respectively.

\subsection{Phenolic compounds}

Phenolic compounds of stem, leaf and seed extracts from $P$. terebinthus were determined and the results are presented in 
Table 1 . The presence of 15 different phenolic compounds was quantified (Fig. 8). Major phenolic compounds of leaf extract were recorded as epicatechin, sinapic acid, gallic acid and benzoic acid. Main components of stem extract were observed as hydroxybenzoic acid and gallic acid. In seed extract, gallic acid, epicatechin, and catechin were found as main phenolic compounds.

\subsection{Antioxidant activity}

DPPH assay was used to determine the radical scavenging activities of the produced chitosan films incorporated with stem, leaf and seed extracts of $P$. terebinthus along with chitosan control. Antioxidant activities of chitosan control film, chitosanstem film, chitosan-leaf film and chitosan-seed film were measured as $37.21 \pm 2.07 \%, 94.43 \pm 0.13 \%, 93.98 \pm 0.17 \%$ and $95.91 \pm 0.15 \%$ respectively. As is seen, the addition of methanol extracts from $P$. terebinthus' stem, leaf and seed notably improved the antioxidant properties of the blend films. This can be ascribed to already known the high antioxidant activity of $P$. terebinthus due to its hydrogen-donating property and rich phenolic content. ${ }^{17}$ Considering these results, it can be stated that with current analysis, one of the main aims of the present study was achieved as highly antioxidative edible films were produced by successfully incorporating the $P$. terebinthus extracts. Current antioxidant results for the produced films were observed to be higher than previously reported chitosan film with green tea extract ${ }^{14}$ and was found close to chitosan-based film with thyme oil. ${ }^{27}$

\subsection{Antimicrobial efficacy}

The antimicrobial activities of chitosan control, chitosan blend films with three different extracts of $P$. terebinthus was investigated against food borne pathogens. This is the first report on the antimicrobial activities of $P$. terebinthus extracts blended chitosan films against food pathogens. The chitosan blend

Table 1 Phenolic compounds of different parts of $P$. terebinthus ( $\mu \mathrm{g}$ $\mathrm{g}^{-1}$ extract)

\begin{tabular}{llll}
$\begin{array}{l}\text { Phenolic } \\
\text { compounds }\end{array}$ & Leaf $\left(\mu \mathrm{g} \mathrm{g}^{-1}\right)$ & Stem $\left(\mu \mathrm{g} \mathrm{g} \mathrm{g}^{-1}\right)$ & Seed $\left(\mu \mathrm{g} \mathrm{g}^{-1}\right)$ \\
\hline Gallic acid & 4232.69 & 5074.34 & 5841.94 \\
Catechin & - & 4197.40 & 3110.45 \\
Chlorogenic acid & 330.40 & 168.87 & 1745.19 \\
Caffeic acid & 873.88 & - & 1968.65 \\
Hydroxybenzoic & 2630.26 & 5903.19 & - \\
acid & & & \\
Epicatechin & 6104.84 & 324.02 & 3710.97 \\
Syringic acid & 2582.08 & 456.45 & 32.56 \\
Coumaric acid & 1060.47 & - & - \\
Trans-ferrulic acid & - & - & - \\
Sinapic acid & 4280.29 & - & - \\
Benzoic acid & 3838.17 & - & 1776.75 \\
Hesperidin & 672.34 & - & - \\
Rosmarinic acid & 1417.50 & - & - \\
Cinnamic acid & 748.95 & 914.59 & - \\
Quercetin & - & - &
\end{tabular}
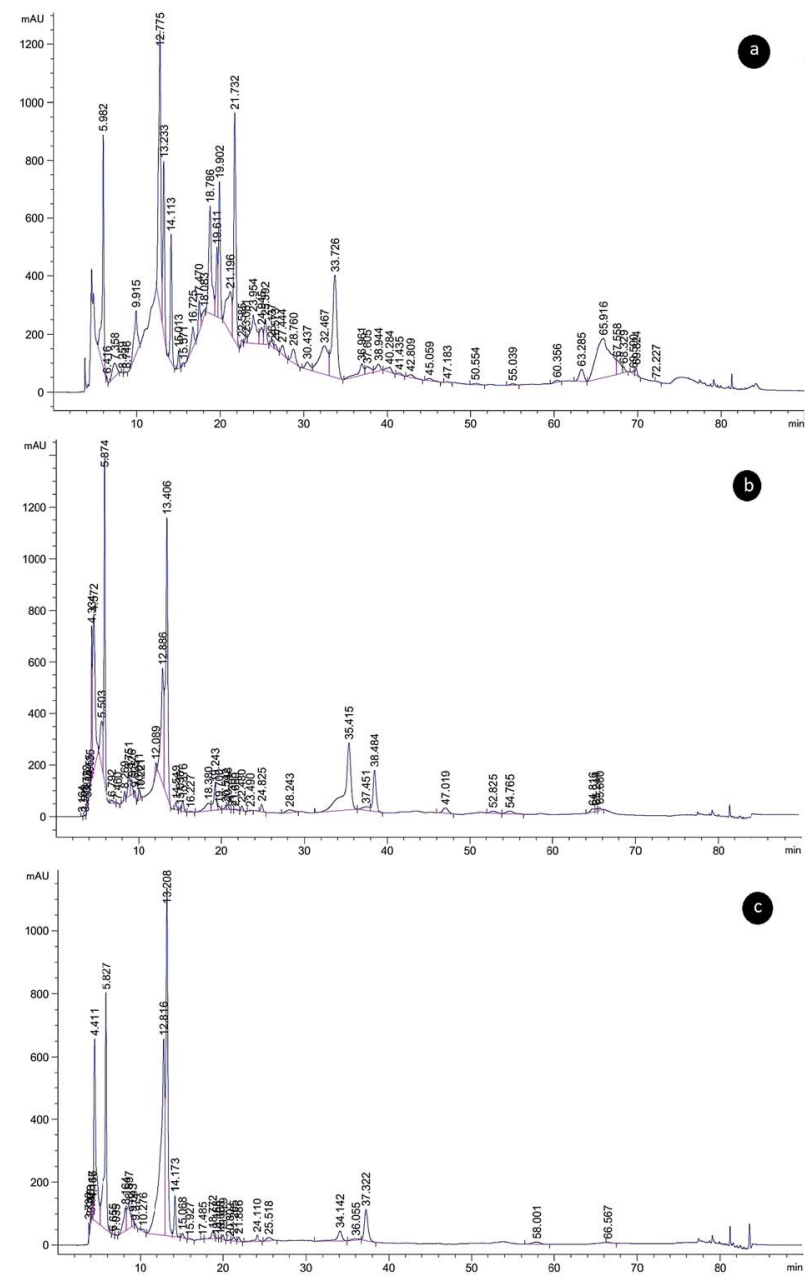

Fig. 8 Chromatograms of the phenolic compounds identified and separated from Pistacia terebinthus' extracts; (a) leaf, (b) seed, (c) stem.

films with the plant extract were found to be effective against especially Gram-negative bacteria such as P. microbilis, P. vulgaris, $P$. aeruginosa and $E$. coli (inhibition zones were ranged in $28.19 \pm 0.92-21.33 \pm 0.74 \mathrm{~mm}$ ). Gram-negative bacteria are often resistant to conventional antimicrobial drugs because of their tenacious nature ability and expression of active efflux pumps however in the current study, Gram-negative bacteria did not reveal any activity in the case of chitosan blended films with $P$. terebinthus' extracts. $^{44,45}$ Furthermore, different compounds of $P$. terebinthus incorporated into chitosan films also presented antibacterial zones as 26.63 \pm 0.81-20.03 \pm $0.68 \mathrm{~mm}$ against Gram positives. Also, chitosan-seed film indicated the greatest antimicrobial activity (ranged in $28.19 \pm$ $0.98-20.33 \pm 0.72 \mathrm{~mm}$ ) than the other blended films. In all tests, control disc with the only solvent displayed no inhibition zone and chitosan control film showed lower inhibition zone than blended films. From this result, it was concluded that incorporating of the plant extract into chitosan films provided higher antimicrobial activity because of compounds of $P$. terebinthus contain several volatile ingredients, most important of which are flavonoids and polyphenols. ${ }^{46,47}$ In addition, the inhibition zones of chitosan blend films indicated higher activity than that 


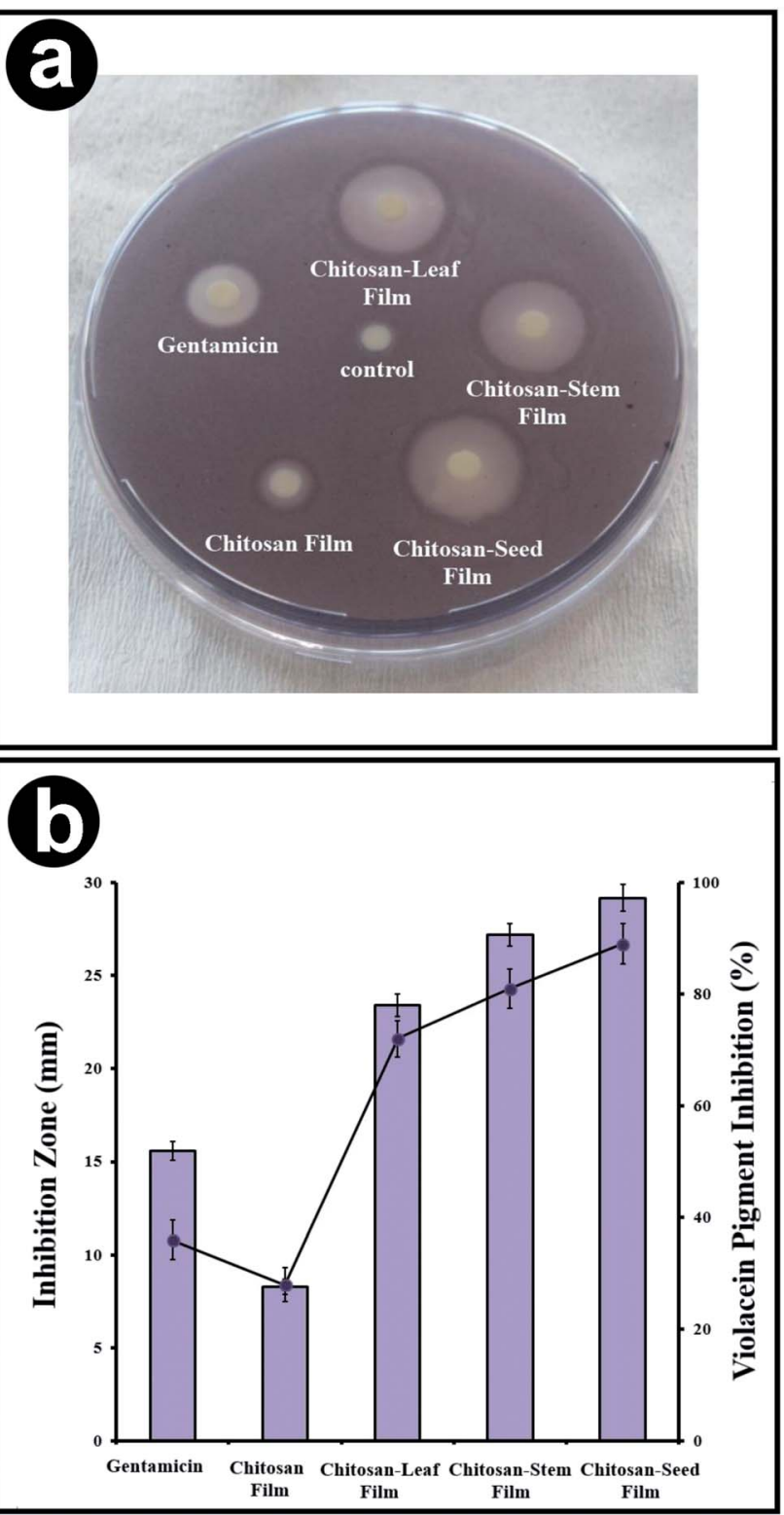

Fig. 9 (a) Plate diffusion assay for the quorum sensing inhibition, (b) inhibition zone $(\mathrm{mm})$ (purple-grey bars) of disc diffusion test and quantitative determination of violacein inhibition (\%) (purple circles) of chitosan films with/without seed, stem and leaf compound extract of Pistacia terebinthus.

of commercial antibiotic (gentamicin). To the most important goal of our study was that to incorporate the plant extract which is rich in flavonoids compounds into the chitosan matrix which has antimicrobial property can efficiently eliminate the persistent microorganisms such as S. typhimurium, E. coli, B. thuringiensis, $P$. aeruginosa and $P$. vulgaris. The discovery of plant and biopolymer based nontoxic agents could promote antimicrobial activity with minimal drawbacks since enhanced toxicity due to the application of conventional toxic chemicals.

\subsection{QSI bioassay}

In the current study, chitosan films blended with extracts of stem, leaf, and seed from P. terebinthus was assessed for the
anti-QS activity against the reporter strain C. violaceum CV026. In the disc diffusion method, the tested chitosan films displayed significant values of anti-QS activity which was computed according to the loss of purple pigmentation. All synthesized films inhibited the production of violacein with zones ranged in 8.11 and $29.38 \mathrm{~mm}$. According to radios of inhibition zones, chitosan blend films with stem, leaf and seed extracts of $P$. terebinthus displayed higher pigment clearance than chitosan control film. Furthermore, interestingly the pigment inhibition of chitosan blended films, especially with seed, showed greater activity than commercial antibiotic (gentamicin). The highest values of quorum-sensing activity were obtained from chitosan-seed film.

On the other hand, quantitative determination of the violacein production inhibition was also evaluated. All the tested chitosan blend films exhibited a significant reduction in production of violacein with the ranged in $28.13 \pm 2.4-89.34 \pm$ $3.5 \%$ (Fig. 9). Similarly, with results of QS inhibition zone, chitosan-seed and chitosan-stem films exerted a higher pigment inhibition ( $89 \pm 3.6$ and $81 \pm 3.5 \%$, respectively) against $C$. violaceum CV026 than chitosan-leaf film $(72 \pm 3.3 \%)$.

\section{Conclusion}

For the first time, stem, leaf and seed extracts obtained from $P$. terebinthus containing high phenolic substances were transferred into chitosan films. As a result, high antioxidative and antimicrobial films (against nine food-borne pathogens) were successfully produced and well characterized with commonly used analytical techniques. Additionally, supplementing plant extracts into chitosan film enhanced the overall elasticity of chitosan-stem and chitosan-seed films. Current results showed that the produced blend films could be suggested as a suitable alternative to synthetic plastics for applications in food coating and packaging industry.

\section{Conflicts of interest}

The authors declare no conflicts of interest.

\section{References}

1 J. G. Derraik, Mar. Pollut. Bull., 2002, 44, 842-852.

2 D. K. Barnes, F. Galgani, R. C. Thompson and M. Barlaz, Philos. Trans. R. Soc. London, Ser. B, 2009, 364, 1985-1998.

3 F. Debeaufort, J.-A. Quezada-Gallo and A. Voilley, Crit. Rev. Food Sci. Nutr., 1998, 38, 299-313.

4 J. M. Krochta, Protein-Based Films Coat., 2002, 1-41.

5 R. A. Talja, H. Helén, Y. H. Roos and K. Jouppila, Carbohydr. Polym., 2008, 71, 269-276.

6 M. Mujtaba, A. M. Salaberria, M. A. Andres, M. Kaya, A. Gunyakti and J. Labidi, Int. J. Biol. Macromol., 2017, 104, 944-952.

7 M. Kaya, L. Akyuz, I. Sargin, M. Mujtaba, A. M. Salaberria, J. Labidi, Y. S. Cakmak, B. Koc, T. Baran and T. Ceter, J. Ind. Eng. Chem., 2017, 47, 236-245.

8 T. Bourtoom, Int. Food Res. J., 2008, 15, 237-248. 
9 R. Avena-Bustillos and J. Krochta, J. Food Sci., 1993, 58, 904907.

10 M. Z. Elsabee and E. S. Abdou, Mater. Sci. Eng., C, 2013, 33, 1819-1841.

11 M. N. R. Kumar, React. Funct. Polym., 2000, 46, 1-27.

12 J. V. Tezotto-Uliana, G. P. Fargoni, G. M. Geerdink and R. A. Kluge, Postharvest Biol. Technol., 2014, 91, 72-77.

13 A. Anitha, S. Sowmya, P. S. Kumar, S. Deepthi, K. Chennazhi, H. Ehrlich, M. Tsurkan and R. Jayakumar, Prog. Polym. Sci., 2014, 39, 1644-1667.

14 U. Siripatrawan and B. R. Harte, Food Hydrocolloids, 2010, 24, 770-775.

15 V. A. Pereira, I. N. Q. de Arruda and R. Stefani, Food Hydrocolloids, 2015, 43, 180-188.

16 H. El Gharras, Int. J. Food Sci. Technol., 2009, 44, 2512-2518.

17 D. D. Kavak, E. Altıok, O. Bayraktar and S. Ülkü, J. Mol. Catal. B: Enzym., 2010, 64, 167-171.

18 A. Ghasemzadeh and N. Ghasemzadeh, J. Med. Plants Res., 2011, 5, 6697-6703.

19 J. Rios and M. Recio, J. Ethnopharmacol., 2005, 100, 80-84.

20 W. X. Du, C. Olsen, R. Avena-Bustillos, M. Friedman and T. McHugh, J. Food Sci., 2011, 76, M149-M155.

21 F. Caponio, V. Alloggio and T. Gomes, Food Chem., 1999, 64, 203-209.

22 J. Choo, Y. Rukayadi and J. K. Hwang, Lett. Appl. Microbiol., 2006, 42, 637-641.

23 H. Schulz and M. Baranska, Vib. Spectrosc., 2007, 43, 13-25.

24 P. Mayachiew and S. Devahastin, Food Chem., 2010, 118, 594-601.

25 B. Dou, V. Dupont, P. T. Williams, H. Chen and Y. Ding, Bioresour. Technol., 2009, 100, 2613-2620.

26 J. Zawadzki and H. Kaczmarek, Carbohydr. Polym., 2010, 80, 394-400.

27 D. Altiok, E. Altiok and F. Tihminlioglu, J. Mater. Sci.: Mater. Med., 2010, 21, 2227-2236.

28 F. M. Pelissari, M. V. Grossmann, F. Yamashita and E. A. G. Pineda, J. Agric. Food Chem., 2009, 57, 7499-7504.

29 P. Kanmani and J.-W. Rhim, Int. J. Biol. Macromol., 2014, 68, 258-266.

30 Y. Peng, Y. Wu and Y. Li, Int. J. Biol. Macromol., 2013, 59, 282-289.
31 J. T. Martins, M. A. Cerqueira and A. A. Vicente, Food Hydrocolloids, 2012, 27, 220-227.

32 S. Shojaee-Aliabadi, H. Hosseini, M. A. Mohammadifar, A. Mohammadi, M. Ghasemlou, S. M. Ojagh, S. M. Hosseini and R. Khaksar, Int. J. Biol. Macromol., 2013, 52, 116-124.

33 M. Ghasemlou, N. Aliheidari, R. Fahmi, S. Shojaee-Aliabadi, B. Keshavarz, M. J. Cran and R. Khaksar, Carbohydr. Polym., 2013, 98, 1117-1126.

34 J. F. Rubilar, R. M. Cruz, H. D. Silva, A. A. Vicente, I. Khmelinskii and M. C. Vieira, J. Food Eng., 2013, 115, 466-474.

35 M. Kaya, P. Ravikumar, S. Ilk, M. Mujtaba, L. Akyuz, J. Labidi, A. M. Salaberria, Y. S. Cakmak and S. K. Erkul, Innovative Food Sci. Emerging Technol., 2017, 45, 287-297.

36 L. Wang, Y. Dong, H. Men, J. Tong and J. Zhou, Food Hydrocolloids, 2013, 32, 35-41.

37 J. Jin, M. Song and D. Hourston, Biomacromolecules, 2004, 5, 162-168.

38 C. Ramírez, I. Gallegos, M. Ihl and V. Bifani, J. Food Eng., 2012, 109, 424-429.

39 U. Siripatrawan and W. Vitchayakitti, Food Hydrocolloids, 2016, 61, 695-702.

40 J. S. Smith and Y. H. Hui, Food processing: principles and applications, John Wiley \& Sons, 2008.

41 M. Hosseini, S. Razavi and M. Mousavi, J. Food Process. Preserv., 2009, 33, 727-743.

42 M. Moradi, H. Tajik, S. M. R. Rohani, A. R. Oromiehie, H. Malekinejad, J. Aliakbarlu and M. Hadian, LWT-Food Sci. Technol., 2012, 46, 477-484.

43 S.-I. Park and Y. Zhao, J. Agric. Food Chem., 2004, 52, 19331939.

44 J.-M. Pagès and L. Amaral, Biochim. Biophys. Acta, Proteins Proteomics, 2009, 1794, 826-833.

45 L. J. Piddock, Clin. Microbiol. Rev., 2006, 19, 382-402.

46 S. Kawashty, S. Mosharrafa, M. El-Gibali and N. Saleh, Biochem. Syst. Ecol., 2000, 28, 915-917.

47 X. Zhao, H. Sun, A. Hou, Q. Zhao, T. Wei and W. Xin, Biochim. Biophys. Acta, Gen. Subj., 2005, 1725, 103-110. 